RAL-TR-98-041

hep-ph/9805413

\title{
The Jet Multiplicity as a Function of Thrust
}

\author{
D. J. Miller \\ and \\ Michael H. Seymour \\ Rutherford Appleton Laboratory, Chilton, \\ Didcot, Oxfordshire. OX11 0QX. England.
}

\begin{abstract}
We calculate the average multiplicity of jets in $e^{+} e^{-}$annihilation as a function of both the jet resolution parameter, $y_{\text {cut }}$, and the thrust $T$. Our result resums to all orders the leading and next-to-leading logarithms in $y_{\text {cut }}$ and $1-T$, and is exact up to second order in $\alpha_{\mathrm{S}}$. This allows a comparison between the number of jets found using jet algorithms and the ability to distinguish different jet topologies via thrust.
\end{abstract}

RAL-TR-98-041

May 1998 


\section{Introduction}

The study of jets and their physics in $\mathrm{e}^{+} \mathrm{e}^{-}$annihilation has added much to our understanding of perturbative QCD. Although hadrons are the final state particles seen in such collisions, it is quarks and gluons whose dynamics are described by perturbative QCD. The definition of jets, via infrared and collinear safe jet clustering algorithms, bridges the gap between the theoretically accessible partons and the experimentally observed hadrons.

The multiplicity of jets in $\mathrm{e}^{+} \mathrm{e}^{-}$annihilation events provides a good example of this. By contrasting the jet multiplicity with the hadron multiplicity we may see some of the advantages of using jets as our object of study. In particular, when predicting the number of hadrons in the final state, one must invoke some non-perturbative hadronization model at an unknown hadronization scale $Q_{0}$. This results in an arbitrary overall normalisation, so that only the energy variation of the hadron multiplicity can be calculated within perturbative QCD. In contrast, by examining the jet multiplicity, the hadronization scale is replaced by the known jet resolution scale $Q_{0}=Q \sqrt{y_{\text {cut }}}$, and no arbitrary parameters are introduced. Therefore, the jet multiplicity is fully calculable within perturbative QCD, including its absolute value. In fact, since the jet resolution scale is firmly within our control it can be varied at will, effectively studying the energy dependence of the multiplicity in a single experiment.

For large values of the jet resolution scale, the jet multiplicity is reliably predicted by fixed-order perturbation theory. However, the appearance of logarithms of $y_{\text {cut }}$ spoils the perturbative expansion for finer jet resolutions and these logarithms must be summed to all orders. With the advent of the $k_{\perp}$ (or Durham) jet finding algorithm[1], it is possible to sum the leading and next-to-leading logarithms of $y_{\text {cut }}$ to all orders, making the jet multiplicity also reliable for small resolution scales [2]. This is the jet algorithm that we will use throughout this paper, and it defines jets according to the following iterative procedure:

- For each pair of particles calculate a separation defined by,

$$
y_{i j}=2 \frac{\min \left(E_{i}^{2}, E_{j}^{2}\right)}{Q^{2}}\left(1-\cos \theta_{i j}\right),
$$

where $E_{i}$ and $E_{j}$ are the particles' energies and $\theta_{i j}$ is the angle between their momenta.

- For the pair of particles with the smallest separation, $y_{i j}$, combine their momenta, $p_{i}$ and $p_{j}$, together to form a pseudo-particle of momentum $p_{i j}$. In the $E$-scheme, $p_{i j}=p_{i}+p_{j}$.

- Repeat the above two steps until all the separations of particles or pseudo-particles are less than the jet resolution parameter $y_{\text {cut }}$. The remaining particles and pseudoparticles are then called jets.

This algorithm can be applied to form jets from either partons (for theoretical considerations) or hadrons (for experimental analysis). The difference between performing the 
algorithm at the partonic and hadronic levels is expected to be suppressed by at least one power of $1 / Q$ [3].

The theoretical calculation and experimental analysis of event shapes have also contributed greatly to our understanding of perturbative QCD. In particular, the thrust and heavy jet mass of an event have been extensively studied[四] and show good agreement with experiment [5]. It is interesting to calculate the multiplicity of jets in $\mathrm{e}^{+} \mathrm{e}^{-}$ annihilation, retaining the jet kinematics so that the multiplicity can be expressed for specific values of one of these event shapes. In this paper we calculate the multiplicity of jets in $\mathrm{e}^{+} \mathrm{e}^{-}$annihilation as a function of the thrust, summing all large logarithms to next-to-leading logarithmic accuracy. This was done for the multiplicity of hadrons in Ref. [6]. The thrust for an event is defined by,

$$
T=\max \left\{\frac{\Sigma_{i}\left|\vec{P}_{i} \cdot \vec{n}\right|}{\Sigma_{i}\left|\vec{P}_{i}\right|}\right\},
$$

where $\vec{n}$ is a unit vector chosen to maximise the right-hand-side, and $\vec{P}_{i}$ are the threemomenta of the final state partons or hadrons. Of course, our results can be easily applied to other similar observables, such as the heavy jet mass.

The structure of the paper will be as follows. In section 2 we shall consider the fixed-order contribution to the multiplicity up to $\mathcal{O}\left(\alpha_{\mathrm{S}}^{2}\right)$. Then, in section 3 , we will resum large logarithms of $\tau \equiv 1-T$ and $y_{\text {cut }}$ to all orders in $\alpha_{\mathrm{S}}$, and to next-to-leading logarithmic accuracy, using the coherent branching formalism. To avoid double counting, we will discuss the matching of this result to fixed order in section 4, and finally, we present numerical results in section 5 .

\section{Fixed Order}

We begin by defining the quantity $N\left(\tau, y_{\text {cut }}\right)$ as the multiplicity of subjets resolved in the $k_{\perp}$ algorithm at a scale $y_{\text {cut }}$, in events with thrust greater than $1-\tau$. It is generally given by,

$$
N\left(\tau, y_{\text {cut }}\right)=\sum_{n=2}^{\infty} \frac{n \sigma_{n}\left(\tau, y_{\text {cut }}\right)}{\sigma_{\text {tot }}}
$$

where $\sigma_{n}\left(\tau, y_{\text {cut }}\right)$ is the cross section for events with thrust greater than $1-\tau$ and exactly $n$ jets, and $\sigma_{\text {tot }}$ is the total cross section for the production of hadrons. We will require this up to second order in the strong coupling constant, $\alpha_{\mathrm{S}}$. Defining $\sigma_{n}^{(m)}\left(\tau, y_{\text {cut }}\right)$ to be the $\mathcal{O}\left(\alpha_{\mathrm{S}}^{m}\right)$ contribution to $\sigma_{n}\left(\tau, y_{\text {cut }}\right)$, the multiplicity to $\mathcal{O}\left(\alpha_{\mathrm{S}}^{2}\right)$ is given by,

$$
\begin{aligned}
& N\left(\tau, y_{\text {cut }}\right)= \\
& \quad \frac{2\left(\sigma_{0}+\sigma_{2}^{(1)}\left(\tau, y_{\text {cut }}\right)+\sigma_{2}^{(2)}\left(\tau, y_{\text {cut }}\right)\right)+3\left(\sigma_{3}^{(1)}\left(\tau, y_{\text {cut }}\right)+\sigma_{3}^{(2)}\left(\tau, y_{\text {cut }}\right)\right)+4 \sigma_{4}^{(2)}\left(\tau, y_{\text {cut }}\right)}{\sigma_{0}+\frac{\alpha_{\mathrm{S}}}{\pi} \sigma_{0}+\left(\frac{\alpha_{\mathrm{S}}}{\pi}\right)^{2} C \sigma_{0}},
\end{aligned}
$$


where $\sigma_{0}$ is the Born cross-section and $C$ is some number that we will not eventually require. Truncating at $\mathcal{O}\left(\alpha_{\mathrm{S}}^{2}\right)$, we have:

$$
\begin{aligned}
N\left(\tau, y_{\text {cut }}\right)=2 & {\left[1-\frac{\alpha_{\mathrm{S}}}{\pi}-C\left(\frac{\alpha_{\mathrm{S}}}{\pi}\right)^{2}+\left(1-\frac{\alpha_{\mathrm{S}}}{\pi}\right) \frac{1}{\sigma_{0}} \sigma_{2}^{(1)}\left(\tau, y_{\text {cut }}\right)+\frac{1}{\sigma_{0}} \sigma_{2}^{(2)}\left(\tau, y_{\text {cut }}\right)+\left(\frac{\alpha_{\mathrm{S}}}{\pi}\right)^{2}\right] } \\
& +3\left(\left(1-\frac{\alpha_{\mathrm{S}}}{\pi}\right) \frac{1}{\sigma_{0}} \sigma_{3}^{(1)}\left(\tau, y_{\text {cut }}\right)+\frac{1}{\sigma_{0}} \sigma_{3}^{(2)}\left(\tau, y_{\text {cut }}\right)\right)+4 \frac{1}{\sigma_{0}} \sigma_{4}^{(2)}\left(\tau, y_{\text {cut }}\right) .
\end{aligned}
$$

Since at $\mathcal{O}\left(\alpha_{\mathrm{S}}\right)$ every event has either 2 or 3 jets and at $\mathcal{O}\left(\alpha_{\mathrm{S}}^{2}\right)$ every event has 2 , 3 or 4 jets, we can write,

$$
\begin{aligned}
\sigma^{(1)}(\tau) & =\sigma_{2}^{(1)}\left(\tau, y_{\text {cut }}\right)+\sigma_{3}^{(1)}\left(\tau, y_{\text {cut }}\right) \\
\sigma^{(2)}(\tau) & =\sigma_{2}^{(2)}\left(\tau, y_{\text {cut }}\right)+\sigma_{3}^{(2)}\left(\tau, y_{\text {cut }}\right)+\sigma_{4}^{(2)}\left(\tau, y_{\text {cut }}\right) .
\end{aligned}
$$

This allows us to write the multiplicity in terms of more inclusive quantities. Further simplification is made by rewriting our expression in terms of the cross section for events with thrust less than $1-\tau, \bar{\sigma}(\tau)$, using,

$$
\begin{gathered}
\sigma^{(1)}(\tau)+\bar{\sigma}^{(1)}(\tau)=\left(\frac{\alpha_{\mathrm{S}}}{\pi}\right) \sigma_{0}, \\
\sigma^{(2)}(\tau)+\bar{\sigma}^{(2)}(\tau)=\left(\frac{\alpha_{\mathrm{S}}}{\pi}\right)^{2} C \sigma_{0} .
\end{gathered}
$$

This gives,

$$
\begin{aligned}
N\left(\tau, y_{\text {cut }}\right)= & 2\left(1-\left(1-\frac{\alpha_{\mathrm{S}}}{\pi}\right) \frac{1}{\sigma_{0}} \bar{\sigma}^{(1)}(\tau)-\frac{1}{\sigma_{0}} \bar{\sigma}^{(2)}(\tau)\right) \\
& +\left(\left(1-\frac{\alpha_{\mathrm{S}}}{\pi}\right) \frac{1}{\sigma_{0}} \sigma_{3}^{(1)}\left(\tau, y_{\text {cut }}\right)+\frac{1}{\sigma_{0}} \sigma_{3}^{(2)}\left(\tau, y_{\text {cut }}\right)\right)+2 \frac{1}{\sigma_{0}} \sigma_{4}^{(2)}\left(\tau, y_{\text {cut }}\right) \\
= & 2(1-R(\tau))+R_{3}\left(\tau, y_{\text {cut }}\right)+2 R_{4}\left(\tau, y_{\text {cut }}\right),
\end{aligned}
$$

where $R(\tau)$ is the ratio of the cross section for events with thrust less than $1-\tau$ to the total cross section, and $R_{n}\left(\tau, y_{\text {cut }}\right)$ is the ratio for events with $n$ jets and thrust greater than $1-\tau$, evaluated to the appropriate order in $\alpha_{\mathrm{S}}$, i.e.

$$
R(\tau) \equiv \frac{1}{\sigma_{\text {tot }}} \bar{\sigma}(\tau), \quad R_{n}\left(\tau, y_{\text {cut }}\right) \equiv \frac{1}{\sigma_{\text {tot }}} \sigma_{n}\left(\tau, y_{\text {cut }}\right)
$$

\subsection{Zeroth Order}

At zeroth order, $\mathcal{O}\left(\alpha_{\mathrm{S}}^{0}\right)$, i.e. with no gluon emission, the cross section is given by the production of a quark-antiquark pair, emitted back-to-back in the centre of mass frame. The quark and antiquark will always be placed in separate jets, so the jet multiplicity will be two, and the thrust axis will fall along their common axis, trivially giving a thrust of one. Therefore we have,

$$
N\left(\tau, y_{\text {cut }}\right)=2 .
$$




\subsection{First Order}

We easily obtain the multiplicity to $\mathcal{O}\left(\alpha_{\mathrm{S}}\right)$ by truncating Eq. (10), giving,

$$
N\left(\tau, y_{\text {cut }}\right)=2(1-R(\tau))+R_{3}\left(\tau, y_{\text {cut }}\right) \text {. }
$$

Since neither of these cross sections involve the singular region of thrust equal to 1, they can be calculated in four dimensions. At this order, i.e. $\mathcal{O}\left(\alpha_{\mathrm{S}}\right)$, the only contribution is from the partonic process $\mathrm{e}^{+} \mathrm{e}^{-} \rightarrow \mathrm{q} \overline{\mathrm{q}} \mathrm{g}$.

For $R(\tau)$, the cross section for producing events with thrust greater than $1-\tau$ divided by the total cross section to hadrons, one obtains,

$$
\begin{aligned}
R(\tau)=\frac{C_{F} \alpha_{\mathrm{S}}}{2 \pi} & \left\{2 \log ^{2} \tau+3 \log \tau+\frac{5}{2}-\frac{\pi^{2}}{3}\right. \\
& -6 \tau \log \tau-4 \log (1-\tau) \log \tau-6 \tau-3(1-2 \tau) \log (1-2 \tau) \\
& \left.+4 \operatorname{Li}_{2}\left(\frac{\tau}{1-\tau}\right)-\frac{9}{2} \tau^{2}+2 \log ^{2}(1-\tau)\right\},
\end{aligned}
$$

where only the first line contributes as $\tau \rightarrow 0$.

Next we turn to $R_{3}\left(\tau, y_{\text {cut }}\right)$, the cross section for producing three jets with thrust greater than $1-\tau$ divided by the total cross section to hadrons. To this order,

$$
R_{3}\left(\tau, y_{\text {cut }}\right)=\frac{1}{\sigma_{0}} \sigma_{3}^{(1)}\left(\tau, y_{\text {cut }}\right)
$$

The contributing partonic process is still $\mathrm{e}^{+} \mathrm{e}^{-} \rightarrow \mathrm{q} \overline{\mathrm{q} g}$, but now we demand that the parton separations be greater than $y_{\text {cut }}$ in order to maintain a three jet final state.

For $y_{\text {cut }}<\frac{2 \tau^{2}}{1-\tau}$ we have,

$$
\begin{aligned}
R_{3}\left(\tau, y_{\text {cut }}\right)= & \frac{C_{F} \alpha_{\mathrm{S}}}{2 \pi}\left\{\int_{1-\tau}^{1-y_{\mathrm{join}}} d x_{1} \int_{1-x_{1} / 2}^{x_{1}} d x_{2}\right. \\
& \left.+\int_{1-y_{\mathrm{join}}}^{1-y_{\text {cut }}} d x_{1} \int_{1-x_{1} / 2}^{\frac{\left(1-x_{1}\right)\left(2-x_{2}\right)}{1+y_{\mathrm{cut}}-x_{1}}} d x_{2}\right\} \frac{2\left(x_{1}^{3}+x_{2}^{3}+\left(2-x_{1}-x_{2}\right)^{3}\right)}{\left(1-x_{1}\right)\left(1-x_{2}\right)\left(x_{1}+x_{2}-1\right)} \\
= & R_{D}\left(y_{\text {cut }}\right)-R(\tau),
\end{aligned}
$$

where,

$$
y_{\text {join }} \equiv \frac{\sqrt{y_{\text {cut }}\left(8+y_{\text {cut }}\right)}-y_{\text {cut }}}{4},
$$

and $R_{D}\left(y_{\text {cut }}\right)$ is the three-jet fraction in the $k_{\perp}$ algorithm at a scale $y_{\text {cut }}$, given in Ref. [7].

For $y_{\text {cut }}>\frac{2 \tau^{2}}{1-\tau}$ this becomes,

$$
\begin{aligned}
R_{3}\left(\tau, y_{\text {cut }}\right) & =\frac{C_{F} \alpha_{\mathrm{S}}}{2 \pi} \int_{1-\tau}^{1-y_{\mathrm{cut}}} d x_{1} \int_{1-x_{1} / 2}^{\frac{\left(1-x_{1}\right)\left(2-x_{2}\right)}{1+y_{\mathrm{cut}}-x_{1}}} d x_{2} \frac{2\left(x_{1}^{3}+x_{2}^{3}+\left(2-x_{1}-x_{2}\right)^{3}\right)}{\left(1-x_{1}\right)\left(1-x_{2}\right)\left(x_{1}+x_{2}-1\right)} \\
& =\frac{C_{F} \alpha_{\mathrm{S}}}{2 \pi} \int_{1-\tau}^{1-y_{\mathrm{cut}}} d x_{1} \frac{g\left(x_{1}, y_{\mathrm{cut}}\right)}{1-x_{1}}
\end{aligned}
$$


with

$$
g\left(x_{1}, y_{\text {cut }}\right)=\frac{2}{x_{1}}\left(2-3 x_{1}\left(1-x_{1}\right)\right) \log \frac{\left(1-x_{1}\right)\left(1-y_{\text {cut }}\right)}{y_{\text {cut }}-\left(1-x_{1}\right)^{2}}-3\left(2-x_{1}\right)^{2} \frac{1-y_{\text {cut }}-x_{1}}{1+y_{\text {cut }}-x_{1}} .
$$

This integral has a closed analytic form, but does not give a very compact expression, so we do not reproduce it here.

In order to match our resummed result to fixed order we must also extract the logarithmic behaviour for $\tau, y_{\text {cut }} \ll 1$. We obtain:

$$
R_{3}\left(\tau, y_{\text {cut }}\right)=\frac{C_{F} \alpha_{\mathrm{S}}}{2 \pi}\left\{\begin{array}{ll}
2 \log ^{2} \frac{\tau}{y_{\text {cut }}}-3 \log \frac{\tau}{y_{\text {cut }}}+\log 64 & y_{\text {cut }}>2 \tau^{2} \\
\log ^{2} \frac{1}{y_{\text {cut }}}-2 \log ^{2} \frac{1}{\tau}-3 \log \frac{\tau}{y_{\text {cut }}}+\log 64+\log ^{2} 2 & y_{\text {cut }}<2 \tau^{2}
\end{array} .\right.
$$

Note that these two solutions are continuous at the matching point, and that it can be shifted, with logarithmic accuracy, to:

$$
R_{3}\left(\tau, y_{\text {cut }}\right)=\frac{C_{F} \alpha_{\mathrm{S}}}{2 \pi} \begin{cases}2 \log ^{2} \frac{\tau}{y_{\text {cut }}}-3 \log \frac{\tau}{y_{\text {cut }}}+\log 64 & y_{\text {cut }}>\tau^{2} \\ \log ^{2} \frac{1}{y_{\text {cut }}}-2 \log ^{2} \frac{1}{\tau}-3 \log \frac{\tau}{y_{\text {cut }}}+\log 64 & y_{\text {cut }}<\tau^{2}\end{cases}
$$

or:

$$
R_{3}\left(\tau, y_{\text {cut }}\right)=\frac{C_{F} \alpha_{S}}{2 \pi}\left\{2 \log ^{2} \frac{\tau}{y_{\text {cut }}}-3 \log \frac{\tau}{y_{\text {cut }}}-\Theta\left(\tau^{2}-y_{\text {cut }}\right) \log ^{2} \frac{\tau^{2}}{y_{\text {cut }}}\right\}
$$

\subsection{Second Order}

Since we could make the first-order calculation in four dimensions, it is possible to calculate the second-order terms using a standard Monte Carlo NLO program like EVENT2[8].

Now Eq. (10) requires no modification and the multiplicity is,

$$
N\left(\tau, y_{\text {cut }}\right)=2(1-R(\tau))+R_{3}\left(\tau, y_{\text {cut }}\right)+2 R_{4}\left(\tau, y_{\text {cut }}\right),
$$

where $R(\tau)$ and $R_{3}\left(\tau, y_{\text {cut }}\right)$ should now be evaluated to NLO, and $R_{4}\left(\tau, y_{\text {cut }}\right)$ to leading order.

Notice that at this order we can study the dependence on the renormalization scale, since we have

$$
R(\tau)=\frac{\alpha_{\mathrm{S}}(\mu)}{2 \pi} A(\tau)+\left(\frac{\alpha_{\mathrm{S}}(\mu)}{2 \pi}\right)^{2}\left(B(\tau)-\frac{3}{2} C_{F} A(\tau)+\frac{b}{2} A(\tau) \log \frac{\mu^{2}}{Q^{2}}\right),
$$

with $b=\frac{11}{3} C_{A}-\frac{2}{3} N_{f}$, and likewise for $R_{3}\left(\tau, y_{\text {cut }}\right)$.

\section{All-Orders Summation of Large Logarithms}

In this section we present the all-orders resummation of all leading $\left(\alpha_{\mathrm{S}}^{n} \log ^{m} \tau \log ^{2 n-m} y_{\text {cut }}\right)$ and next-to-leading $\left(\alpha_{\mathrm{S}}^{n} \log ^{m} \tau \log ^{2 n-m-1} y_{\text {cut }}\right.$ and $\left.\alpha_{\mathrm{S}}^{n} \log ^{m-1} \tau \log ^{2 n-m} y_{\text {cut }}\right)$ logarithms, which appear for small $y_{\text {cut }}$ and/or $\tau$. These logarithms can be summed to all orders using the coherent branching formalism. 
To next-to-leading logarithmic accuracy, we have,

$$
N\left(\tau, y_{\text {cut }}\right)=2 F_{q}\left(\tau Q^{2}, Q^{2}\right) N_{q}\left(\tau Q^{2}, Q^{2} ; y_{\text {cut }} Q^{2}\right) .
$$

Here, $F_{q}\left(k^{2}, Q^{2}\right)$ is the probability that a quark formed at scale $\mathrm{Q}$ has a mass below $k$, and $\log F_{q}$ has been calculated in Ref. [四 to next-to-leading logarithmic accuracy. Here we only require the expression for $F_{q}$ itself to next-to-leading logarithmic accuracy, which is,

$$
F_{q}\left(k^{2}, Q^{2}\right)=\exp \left\{C_{F} \log \left(\frac{Q^{2}}{k^{2}}\right) f_{1}\left(\frac{\alpha_{\mathrm{S}}(Q)}{4 \pi} b \log \left(\frac{Q^{2}}{k^{2}}\right)\right)+\frac{3}{2} C_{F} f_{2}\left(\frac{\alpha_{\mathrm{S}}(Q)}{4 \pi} b \log \left(\frac{Q^{2}}{k^{2}}\right)\right)\right\},
$$

where,

$$
\begin{aligned}
f_{1}(\lambda) & =\frac{2}{b \lambda}\left[(1-2 \lambda) \log \left(\frac{1}{1-2 \lambda}\right)-2(1-\lambda) \log \left(\frac{1}{1-\lambda}\right)\right], \\
f_{2}(\lambda) & =\frac{2}{b} \log \left(\frac{1}{1-\lambda}\right) .
\end{aligned}
$$

Also, $N_{q}\left(k^{2}, Q^{2} ; Q_{0}^{2}\right)$ is the contribution to the multiplicity in a quark jet formed at a scale $Q$ and resolved at a scale $Q_{0}$, from jet masses below $k$.

In order to derive $N_{q}\left(k^{2}, Q^{2} ; Q_{0}^{2}\right)$, let us first consider the quantity $n_{q}^{a}\left(k^{2}, Q^{2} ; Q_{0}^{2}\right) d k^{2}$ which is the multiplicity of partons of species $a$ in the jet, where the squared mass of the jet lies between $k^{2}$ and $k^{2}+d k^{2}$. Clearly,

$$
N_{q}\left(k^{2}, Q^{2} ; Q_{0}^{2}\right)=\sum_{a=\{q, g\}} \int_{0}^{k^{2}} d q^{2} n_{q}^{a}\left(q^{2}, Q^{2} ; Q_{0}^{2}\right) .
$$

This obeys an exclusive evolution equation given by,

$$
\begin{aligned}
n_{q}^{a}\left(k^{2}, Q^{2} ;\right. & \left.Q_{0}^{2}\right)=\Delta\left(Q^{2}\right) \delta_{q a} \delta\left(k^{2}\right) \Theta\left(Q-Q_{0}\right) \\
+ & \Theta\left(Q-Q_{0}\right) \int_{0}^{Q^{2}} \frac{d q^{2}}{q^{2}} \frac{\Delta\left(Q^{2}\right)}{\Delta\left(q^{2}\right)} \int_{0}^{1} d z \frac{\alpha_{\mathrm{S}}(z(1-z) q)}{2 \pi} P_{g q}(z) \int_{0}^{\infty} d k_{q}^{2} \int_{0}^{\infty} d k_{g}^{2} \\
& \left(n_{g}^{a}\left(k_{g}^{2}, z^{2} q^{2}\right) f_{q}\left(k_{q}^{2},(1-z)^{2} q^{2}\right) \Theta\left(z q-Q_{0}\right)\right. \\
& \left.\quad+n_{q}^{a}\left(k_{q}^{2},(1-z)^{2} q^{2} ; Q_{0}^{2}\right) f_{g}\left(k_{g}^{2}, z^{2} q^{2}\right) \Theta\left((1-z) q-Q_{0}\right)\right) \\
& \delta\left(k^{2}-z(1-z) q^{2}-\frac{k_{g}^{2}}{z}-\frac{k_{q}^{2}}{1-z}\right) .
\end{aligned}
$$

This has a simple physical interpretation. Firstly, it is possible that the quark does not emit any radiation. Then only the original quark will contribute to the multiplicity if it is sufficiently hard (i.e. $Q>Q_{0}$ ). Clearly the resulting jet will be massless. This gives rise to the first term. Alternatively, the quark may survive until it reaches a scale $q$ before emitting a gluon of mass $k_{g}$ and light-cone momentum fraction $z$ (and 
leaving the quark with mass $k_{q}$ and light-cone momentum fraction $1-z$ ). Then the resolvable partons originating from both quark and gluon must be counted. The function $f_{q}\left(k^{2}, Q^{2}\right) d k^{2}$ in the above is the probability that a quark formed at a scale $Q$ has a squared mass between $k^{2}$ and $k^{2}+d k^{2}$, i.e. it is the derivative of $F_{q}\left(k^{2}, Q^{2}\right)$ with respect to $k^{2} . \Delta$ is the Sudakov form factor,

$$
\log \Delta\left(Q^{2}\right)=-\int_{0}^{Q^{2}} \frac{d q^{2}}{q^{2}} \int_{0}^{1} d z P_{g q}(z) \frac{\alpha_{\mathrm{S}}(z(1-z) q)}{2 \pi} .
$$

These equations have only formal meaning, because the integrals are divergent and the form factor is zero. To make them physically meaningful, they can be calculated explicitly by imposing an infrared cutoff $z(1-z) q>\epsilon$, or by working in $d=4-2 \epsilon$ dimensions. They are however infrared finite, so the limit $\epsilon \rightarrow 0$ can be taken smoothly. Thus we always imply such a procedure.

Differentiating with respect to $\log Q^{2}$ yields an inclusive evolution equation:

$$
\begin{aligned}
Q^{2} \frac{d}{d Q^{2}} n_{q}^{a}\left(k^{2}, Q^{2} ;\right. & \left.Q_{0}^{2}\right)=\int_{0}^{1} d z \frac{\alpha_{\mathrm{S}}(z(1-z) Q)}{2 \pi} P_{g q}(z)\left\{\int_{0}^{\infty} d k_{q}^{2} \int_{0}^{\infty} d k_{g}^{2}\right. \\
& \left(n_{g}^{a}\left(k_{g}^{2}, z^{2} Q^{2} ; Q_{0}^{2}\right) f_{q}\left(k_{q}^{2},(1-z)^{2} Q^{2}\right) \Theta\left(z^{2} Q^{2}-Q_{0}^{2}\right)\right. \\
& \left.+n_{q}^{a}\left(k_{q}^{2},(1-z)^{2} Q^{2} ; Q_{0}^{2}\right) f_{g}\left(k_{g}^{2}, z^{2} Q^{2}\right) \Theta\left((1-z)^{2} Q^{2}-Q_{0}^{2}\right)\right) \\
& \left.\delta\left(k^{2}-z(1-z) Q^{2}-\frac{k_{g}^{2}}{z}-\frac{k_{q}^{2}}{1-z}\right)-n_{q}^{a}\left(k^{2}, Q^{2} ; Q_{0}^{2}\right) \Theta\left(Q-Q_{0}\right)\right\} .
\end{aligned}
$$

The mass of the gluon will not significantly contribute to the mass of the jet so to nextto-leading logarithmic accuracy it can be omitted from the $\delta$-function. This $\delta$-function and the normalisation conditions,

$$
\begin{aligned}
\int_{0}^{\infty} d k^{2} f_{q}\left(k^{2}, Q^{2}\right) & =1 \\
\int_{0}^{\infty} d k^{2} n_{q}^{a}\left(k^{2}, Q^{2} ; Q_{0}^{2}\right) & =\mathcal{N}_{q}^{a}\left(Q^{2} ; Q_{0}^{2}\right),
\end{aligned}
$$

can then be used to integrate out $k_{q}^{2}$ and $k_{g}^{2}$. Here $\mathcal{N}_{q}^{a}\left(Q^{2} ; Q_{0}^{2}\right)$ is the multiplicity of partons of species $a$ inside a quark jet, where the quark is emitted at a scale $Q$ and the partons are resolved at a scale $Q_{0}$ and no demand is made on the mass of the jet. This has been derived in Ref. [2],

$$
\begin{aligned}
& \mathcal{N}_{q}^{q}\left(Q^{2} ; Q_{0}^{2}\right)=\mathcal{N}^{-}\left(Q^{2} ; Q_{0}^{2}\right)+\frac{8}{3} \frac{C_{F}}{C_{A}} \frac{N_{f}}{b} \widetilde{\mathcal{N}}\left(Q^{2} ; Q_{0}^{2}\right), \\
& \mathcal{N}_{q}^{g}\left(Q^{2} ; Q_{0}^{2}\right)=\mathcal{N}^{+}\left(Q^{2} ; Q_{0}^{2}\right)-\frac{8}{3} \frac{C_{F}}{C_{A}} \frac{N_{f}}{b} \widetilde{\mathcal{N}}\left(Q^{2} ; Q_{0}^{2}\right),
\end{aligned}
$$

where,

$$
\begin{aligned}
\mathcal{N}^{+}\left(Q^{2} ; Q_{0}^{2}\right) & =z_{1}\left(\frac{z_{0}}{z_{1}}\right)^{B}\left[I_{B+1}\left(z_{1}\right) K_{B}\left(z_{0}\right)+K_{B+1}\left(z_{1}\right) I_{B}\left(z_{0}\right)\right] \\
\mathcal{N}^{-}\left(Q^{2} ; Q_{0}^{2}\right) & =\left(\frac{z_{0}}{z_{1}}\right)^{\frac{8}{3} \frac{C_{F}}{C_{A}} \frac{N_{f}}{b}} \\
\widetilde{\mathcal{N}}\left(Q^{2} ; Q_{0}^{2}\right) & =\left(\frac{z_{0}}{z_{1}}\right)^{B}\left[I_{B}\left(z_{1}\right) K_{B}\left(z_{0}\right)-K_{B}\left(z_{1}\right) I_{B}\left(z_{0}\right)\right]
\end{aligned}
$$


and

$$
B=\frac{1}{b}\left(\frac{11}{3} C_{A}+\frac{2 N_{f}}{3}-\frac{4 C_{F} N_{f}}{3 C_{A}}\right) .
$$

$I_{\nu}$ and $K_{\nu}$ are the modified Bessel functions and we have used the notation of Ref. [2] where,

$$
z_{1}^{2}=\frac{32 \pi C_{A}}{b^{2} \alpha_{\mathrm{S}}(Q)}, \quad z_{0}^{2}=\frac{32 \pi C_{A}}{b^{2} \alpha_{\mathrm{S}}\left(Q_{0}\right)} .
$$

Removal of these integrations gives,

$$
\begin{aligned}
& Q^{2} \frac{d}{d Q^{2}} n_{q}^{a}\left(k^{2}, Q^{2} ; Q_{0}^{2}\right)=\int_{0}^{1} d z \frac{\alpha_{\mathrm{S}}(z(1-z) Q)}{2 \pi} P_{g q}(z) \\
& \left(\mathcal{N}_{g}^{a}\left(z^{2} Q^{2} ; Q_{0}^{2}\right) f_{q}\left((1-z)\left(k^{2}-z(1-z) Q^{2}\right),(1-z)^{2} q^{2}\right) \Theta\left(z Q-Q_{0}\right) \Theta\left(k^{2}-z(1-z) Q^{2}\right)\right. \\
& \quad+n_{q}^{a}\left((1-z)\left(k^{2}-z(1-z) Q^{2}\right),(1-z)^{2} Q^{2} ; Q_{0}^{2}\right) \Theta\left((1-z) Q-Q_{0}\right) \Theta\left(k^{2}-z(1-z) Q^{2}\right) \\
& \left.\quad-n_{q}^{a}\left(k^{2}, Q^{2} ; Q_{0}^{2}\right) \Theta\left(Q-Q_{0}\right)\right) .
\end{aligned}
$$

The above evolution is dominated by the emission of soft gluons $(z \approx 0)$, so to next-toleading logarithmic accuracy $z$ can be replaced by zero in any smooth functions. Since neither $f_{q}$ nor $n_{q}^{a}$ are smooth functions (they contain $1 / k^{2}$-like terms) such a replacement cannot be made in their arguments. However, their integrated distributions,

$$
\begin{aligned}
F_{q}\left(k^{2}, Q^{2}\right) & =\int_{0}^{k^{2}} d q^{2} f_{q}\left(q^{2}, Q^{2}\right), \\
N_{q}^{a}\left(k^{2}, Q^{2} ; Q_{0}^{2}\right) & =\int_{0}^{k^{2}} d q^{2} n_{q}^{a}\left(q^{2}, Q^{2} ; Q_{0}^{2}\right),
\end{aligned}
$$

which are the functions we are really interested in, are smooth and obey the same evolution equations as $f_{q}$ and $n_{q}^{a}$ but with different boundary conditions.

Integrating Eq. (40) over $k^{2}$ and replacing $z$ with zero where appropriate gives to the required accuracy,

$$
\begin{aligned}
Q^{2} \frac{d}{d Q^{2}} N_{q}^{a}\left(k^{2}, Q^{2} ; Q_{0}^{2}\right)= & \int_{0}^{1} d z \frac{\alpha_{\mathrm{S}}(z Q)}{2 \pi} P_{g q}(z) \\
& \left(\mathcal{N}_{g}^{a}\left(z^{2} Q^{2} ; Q_{0}^{2}\right) F_{q}\left(k^{2}-z Q^{2}, Q^{2}\right) \Theta\left(z Q-Q_{0}\right) \Theta\left(k^{2}-z Q^{2}\right)\right. \\
& +N_{q}^{a}\left(k^{2}-z Q^{2}, Q^{2} ; Q_{0}^{2}\right) \Theta\left(k^{2}-z Q^{2}\right) \Theta\left(Q-Q_{0}\right) \\
& \left.-N_{q}^{a}\left(k^{2}, Q^{2} ; Q_{0}^{2}\right) \Theta\left(Q-Q_{0}\right)\right) .
\end{aligned}
$$

Furthermore, $k^{2}-z Q^{2}$ can be replaced with $k^{2}$ in $F_{q}$ and $N_{q}^{a}$ to next-to-leading logarithmic accuracy, giving the final evolution equation in integral form,

$$
\begin{aligned}
& N_{q}^{a}\left(k^{2}, Q^{2} ; Q_{0}^{2}\right)=\delta_{q a} \\
& +\int_{0}^{Q^{2}} \frac{d q^{2}}{q^{2}} \int_{0}^{1} d z \frac{\alpha_{\mathrm{S}}(z q)}{2 \pi} P_{g q}(z) F_{q}\left(k^{2}, q^{2}\right) \mathcal{N}_{g}^{a}\left(z^{2} q^{2} ; Q_{0}^{2}\right) \Theta\left(k^{2}-z q^{2}\right) \Theta\left(z q-Q_{0}\right) \\
& -\int_{k^{2}}^{Q^{2}} \frac{d q^{2}}{q^{2}} \int_{k^{2} / q^{2}}^{1} d z \frac{\alpha_{\mathrm{S}}(z q)}{2 \pi} P_{g q}(z) N_{q}^{a}\left(k^{2}, Q^{2} ; Q_{0}^{2}\right) .
\end{aligned}
$$


This has a formal solution:

$$
\begin{aligned}
N_{q}^{a}\left(k^{2}, Q^{2} ; Q_{0}^{2}\right)= & \exp \left\{-\int_{k^{2}}^{Q^{2}} \frac{d q^{2}}{q^{2}} \int_{k^{2} / q^{2}}^{1} d z \frac{\alpha_{\mathrm{S}}(z q)}{2 \pi} P_{g q}(z)\right\}\left\{\mathcal{N}_{q}^{a}\left(k^{2} ; Q_{0}^{2}\right)\right. \\
+ & \int_{0}^{Q^{2}} \frac{d q^{2}}{q^{2}} \exp \left[\int_{k^{2}}^{q^{2}} \frac{d \tilde{q}^{2}}{\tilde{q}^{2}} \int_{k^{2} / \tilde{q}^{2}}^{1} d \bar{z} \frac{\alpha_{\mathrm{S}}(\bar{z} \tilde{q})}{\pi} P_{g q}(\bar{z})\right] F_{q}\left(k^{2}, q^{2}\right) \\
& \left.\times \int_{0}^{1} d z \frac{\alpha_{\mathrm{S}}(z q)}{2 \pi} P_{g q}(z) \mathcal{N}_{g}^{a}\left(z^{2} q^{2} ; Q_{0}^{2}\right) \Theta\left(k^{2}-z q^{2}\right) \Theta\left(z q-Q_{0}\right)\right\} .
\end{aligned}
$$

The integrals are considerably simplified by inserting the unintegrated expression for $F_{q}$ [四],

$$
F_{q}\left(k^{2}, Q^{2}\right)=\exp \left\{-\int_{k^{2}}^{Q^{2}} \frac{d q^{2}}{q^{2}} \int_{k^{2} / q^{2}}^{1} d z \frac{\alpha_{\mathrm{S}}(z q)}{2 \pi} P_{g q}(z)\right\}
$$

so that the expression for $N_{q}^{a}$ becomes,

$$
N_{q}^{a}\left(k^{2}, Q^{2} ; Q_{0}^{2}\right)=F_{q}\left(k^{2}, Q^{2}\right)\left\{\mathcal{N}_{q}^{a}\left(k^{2} ; Q_{0}^{2}\right)+\mathcal{C}_{q}^{a}\left(k^{2}, Q^{2} ; Q_{0}^{2}\right)\right\}
$$

where,

$$
\mathcal{C}_{q}^{a}\left(k^{2}, Q^{2} ; Q_{0}^{2}\right)=\int_{k^{2}}^{Q^{2}} \frac{d q^{2}}{q^{2}} \int_{0}^{1} d z \frac{\alpha_{\mathrm{S}}(z q)}{2 \pi} P_{g q}(z) \mathcal{N}_{g}^{a}\left(z^{2} q^{2} ; Q_{0}^{2}\right) \Theta\left(k^{2}-z q^{2}\right) \Theta\left(z q-Q_{0}\right)
$$

The remaining integral can be done by making a change of variable from $q^{2}$ to $\tilde{q}^{2}=z^{2} q^{2}$. To next-to-leading logarithmic accuracy,

$$
\begin{aligned}
\mathcal{C}_{q}^{a}\left(k^{2}, Q^{2} ; Q_{0}^{2}\right) & =\int_{0}^{k^{4} / Q^{2}} \frac{d \tilde{q}^{2}}{\tilde{q}^{2}} \int_{\tilde{q} / Q}^{\tilde{q} / k} d z \frac{\alpha_{\mathrm{S}}(\tilde{q})}{2 \pi} P_{g q}(z) \mathcal{N}_{g}^{a}\left(\tilde{q}^{2} ; Q_{0}^{2}\right) \Theta\left(\tilde{q}-Q_{0}\right) \\
& +\int_{k^{4} / Q^{2}}^{k^{2}} \frac{d \tilde{q}^{2}}{\tilde{q}^{2}} \int_{\tilde{q}^{2} / Q^{2}}^{\tilde{q} / k} d z \frac{\alpha_{\mathrm{S}}(\tilde{q})}{2 \pi} P_{g q}(z) \mathcal{N}_{g}^{a}\left(\tilde{q}^{2} ; Q_{0}^{2}\right) \Theta\left(\tilde{q}-Q_{0}\right) \\
& =2 C_{F} \int_{0}^{k^{4} / Q^{2}} \frac{d \tilde{q}^{2}}{\tilde{q}^{2}} \frac{\alpha_{\mathrm{S}}(\tilde{q})}{2 \pi} \mathcal{N}_{g}^{a}\left(\tilde{q}^{2} ; Q_{0}^{2}\right) \log \left(\frac{Q}{k}\right) \Theta\left(\tilde{q}-Q_{0}\right) \\
& +2 C_{F} \int_{k^{4} / Q^{2}}^{k^{2}} \frac{d \tilde{q}^{2}}{\tilde{q}^{2}} \frac{\alpha_{\mathrm{S}}(\tilde{q})}{2 \pi} \mathcal{N}_{g}^{a}\left(\tilde{q}^{2} ; Q_{0}^{2}\right) \log \left(\frac{k}{\tilde{q}}\right) \Theta\left(\tilde{q}-Q_{0}\right)
\end{aligned}
$$

Making the $\Theta$-functions more explicit and rearranging gives,

$$
\begin{aligned}
\mathcal{C}_{q}^{a}\left(k^{2}, Q^{2} ; Q_{0}^{2}\right) & =\Theta\left(k^{2}-Q Q_{0}\right) 2 C_{F} \int_{Q_{0}^{2}}^{k^{4} / Q^{2}} \frac{d \tilde{q}^{2}}{\tilde{q}^{2}} \frac{\alpha_{\mathrm{S}}(\tilde{q})}{2 \pi} \mathcal{N}_{g}^{a}\left(\tilde{q}^{2} ; Q_{0}^{2}\right) \log \left(\frac{Q}{k}\right) \\
& +\Theta\left(k^{2}-Q_{0}^{2}\right) 2 C_{F} \int_{Q_{0}^{2}}^{k^{2}} \frac{d \tilde{q}^{2}}{\tilde{q}^{2}} \frac{\alpha_{\mathrm{S}}(\tilde{q})}{2 \pi} \mathcal{N}_{g}^{a}\left(\tilde{q}^{2} ; Q_{0}^{2}\right) \log \left(\frac{k}{\tilde{q}}\right) \\
& -\Theta\left(k^{2}-Q Q_{0}\right) 2 C_{F} \int_{Q_{0}^{2}}^{k^{4} / Q^{2}} \frac{d \tilde{q}^{2}}{\tilde{q}^{2}} \frac{\alpha_{\mathrm{S}}(\tilde{q})}{2 \pi} \mathcal{N}_{g}^{a}\left(\tilde{q}^{2} ; Q_{0}^{2}\right) \log \left(\frac{k}{\tilde{q}}\right) \\
& =C_{F}\left\{I^{a}\left(k^{2} ; Q_{0}^{2}\right)-I^{a}\left(k^{4} / Q^{2} ; Q_{0}^{2}\right)\right\},
\end{aligned}
$$


where

$$
I^{a}\left(k^{2} ; Q_{0}^{2}\right)=\Theta\left(k^{2}-Q_{0}^{2}\right) \int_{Q_{0}^{2}}^{k^{2}} \frac{d q^{2}}{q^{2}} \frac{\alpha_{\mathrm{S}}(q)}{2 \pi} \mathcal{N}_{g}^{a}\left(q^{2} ; Q_{0}^{2}\right) \log \left(\frac{k^{2}}{q^{2}}\right) .
$$

This integral is most easily done using the change of variables, Eq. (39), with the obvious addition,

$$
z_{k}^{2}=\frac{32 \pi C_{A}}{b^{2} \alpha_{\mathrm{S}}(k)}
$$

so that it becomes,

$$
I^{a}\left(k^{2}, Q_{0}^{2}\right)=\Theta\left(z_{k}-z_{0}\right) \frac{1}{2 C_{A}} \int_{z_{0}}^{z_{k}} \frac{d z}{z} \mathcal{N}_{g}^{a}\left(z ; z_{0}\right)\left(z_{k}^{2}-z^{2}\right) .
$$

Using the results,

$$
\begin{aligned}
\int_{z_{0}}^{z_{k}} \frac{d z}{z} \mathcal{N}^{+}\left(z ; z_{0}\right)\left(z_{k}^{2}-z^{2}\right) & =2\left(\mathcal{N}^{+}\left(z_{k} ; z_{0}\right)-1\right)+4 B \widetilde{\mathcal{N}}\left(z_{k} ; z_{0}\right) \\
\int_{z_{0}}^{z_{k}} \frac{d z}{z} \widetilde{\mathcal{N}}\left(z ; z_{0}\right)\left(z_{k}^{2}-z^{2}\right) & =2 \widetilde{\mathcal{N}}\left(z_{k} ; z_{0}\right)-z_{k}^{2} / z_{0}^{2}+1
\end{aligned}
$$

we obtain,

$$
\begin{aligned}
I^{g}\left(k^{2}, Q_{0}^{2}\right)= & \Theta\left(z_{k}-z_{0}\right) \frac{1}{C_{A}}\left[\mathcal{N}^{+}\left(z_{k} ; z_{0}\right)-1+2 B \widetilde{\mathcal{N}}\left(z_{k} ; z_{0}\right)\right. \\
& \left.-\frac{C}{2}\left(2 \widetilde{\mathcal{N}}\left(z_{k} ; z_{0}\right)-z_{k}^{2} / z_{0}^{2}+1\right)\right], \\
I^{q}\left(k^{2}, Q_{0}^{2}\right)= & \Theta\left(z_{k}-z_{0}\right) \frac{C}{2 C_{F}}\left(2 \widetilde{\mathcal{N}}\left(z_{k} ; z_{0}\right)-z_{k}^{2} / z_{0}^{2}+1\right),
\end{aligned}
$$

where the parameters $B$ and $C$ are given by Eq. (38) and

$$
C=\frac{8}{3} \frac{N_{f}}{b} \frac{C_{F}}{C_{A}}
$$

However, the quantity of interest is the multiplicity of all jets, independently of their flavour, i.e. $N_{q}=N_{q}^{q}+N_{q}^{g}$. Therefore we finally have,

$$
N_{q}\left(k^{2}, Q^{2} ; Q_{0}^{2}\right)=F_{q}\left(k^{2}, Q^{2}\right)\left\{\mathcal{N}_{q}\left(k^{2} ; Q_{0}^{2}\right)+C_{F}\left(I\left(k^{2}, Q_{0}^{2}\right)-I\left(k^{4} / Q^{2}, Q_{0}^{2}\right)\right)\right\},
$$

with,

$$
\begin{gathered}
I\left(k^{2}, Q_{0}^{2}\right)=\Theta\left(z_{k}-z_{0}\right) \frac{1}{C_{A}}\left[\mathcal{N}^{+}\left(z_{k} ; z_{0}\right)-1+2 B \widetilde{\mathcal{N}}\left(z_{k} ; z_{0}\right)\right. \\
\left.+(B-1)\left(2 \widetilde{\mathcal{N}}\left(z_{k} ; z_{0}\right)-z_{k}^{2} / z_{0}^{2}+1\right)\right] .
\end{gathered}
$$

The above equation conforms with naïve expectations. One might expect that the number of jets of mass below $k^{2}$ resulting from a quark created at a scale $Q^{2}$ would be simply the probability of finding a jet of mass below $k^{2}$ multiplied by the number of jets within. Indeed, this is the first term of our expression for $N_{q}$, and the naïve expectation requires only the addition of next-to-leading logarithmic corrections. 
For completeness, we also present the result for $N_{g}\left(k^{2}, Q^{2}, Q_{0}^{2}\right)$, the multiplicity of partons found within a gluon of squared mass below $k^{2}$. The derivation closely follows that of the quark case above and will not be reproduced here. For the tagged multiplicities we have,

$$
N_{g}^{a}\left(k^{2}, Q^{2} ; Q_{0}^{2}\right)=F_{g}\left(k^{2}, Q^{2}\right)\left\{\mathcal{N}_{g}^{a}\left(k^{2} ; Q_{0}^{2}\right)+C_{A}\left(I^{a}\left(k^{2}, Q_{0}^{2}\right)-I^{a}\left(k^{4} / Q^{2}, Q_{0}^{2}\right)\right)\right\},
$$

Here $F_{g}\left(k^{2}, Q^{2}\right)$ is the integrated gluon jet mass distribution [4], and takes the same form as $F_{q}$, Eq. (26), but with the coefficients of $f_{1}$ and $f_{2}$ now being $C_{A}$ and $b / 2$ respectively. Also $\mathcal{N}_{g}^{a}\left(k^{2} ; Q_{0}^{2}\right)$ is the multiplicity of parton species $a$ found within the gluon[2].

Notice that the function $I^{a}$ is identical to that in the quark case. This is because the only contribution to these functions is from the singular parts of the splitting kernels in the evolution equation. The appropriate kernel for the quark is $P_{g q}(z)=2 C_{F} / z+\mathcal{O}(1)$ and for the gluon is $P_{g g}(z)=2 C_{A} / z+\mathcal{O}(1)$ (the other kernels $P_{q q}$ and $P_{q g}$ have no singular parts and cannot contribute). The only difference between the two cases is the colour factor, $C_{A}$ or $C_{F}$, which is reflected in the coefficient of $I^{a}$.

Clearly, the untagged multiplicity is given by,

$$
N_{g}\left(k^{2}, Q^{2} ; Q_{0}^{2}\right)=F_{g}\left(k^{2}, Q^{2}\right)\left\{\mathcal{N}_{g}\left(k^{2} ; Q_{0}^{2}\right)+C_{A}\left(I\left(k^{2}, Q_{0}^{2}\right)-I\left(k^{4} / Q^{2}, Q_{0}^{2}\right)\right)\right\} .
$$

\section{Matching to Fixed Order}

Of course, the resummed result also contains part of the fixed-order contribution, which has already been included. It must be matched to fixed order so that we do not overcount. This is done by expanding the resummed result to next-to-leading order in $\alpha_{\mathrm{S}}$, and subtracting the offending piece from our result. Expanding each term individually, we obtain:

$$
\begin{aligned}
F_{q}\left(e^{-L} Q^{2}, Q^{2}\right) \approx & 1-\frac{\alpha_{\mathrm{S}}}{2 \pi}\left(C_{F} L^{2}-\frac{3}{2} C_{F} L\right) \\
& +\left(\frac{\alpha_{\mathrm{S}}}{2 \pi}\right)^{2}\left(\frac{1}{2} C_{F}^{2} L^{4}-\left[\frac{1}{2} b C_{F}+\frac{3}{2} C_{F}^{2}\right] L^{3}+\left[\frac{3}{8} b C_{F}+\frac{9}{8} C_{F}^{2}\right] L^{2}\right),
\end{aligned}
$$

where $\alpha_{\mathrm{S}}=\alpha_{\mathrm{S}}(Q)$,

$$
\begin{aligned}
\mathcal{N}_{q}\left(k^{2} ; e^{-l} k^{2}\right) \approx & 1+\frac{\alpha_{\mathrm{S}}}{2 \pi}\left(\frac{1}{2} C_{F} l^{2}-\frac{3}{2} C_{F} l\right)+\left(\frac{\alpha_{\mathrm{S}}}{2 \pi}\right)^{2}\left(\frac{1}{24} C_{F} C_{A} l^{4}-\frac{1}{12} C_{F}\left[3 C_{A}-b\right] l^{3}\right. \\
& \left.+\frac{2\left(C_{A}-C_{F}\right) C_{F} T_{R} N_{f}\left(8 C_{F} T_{R} N_{f}-2 C_{A} T_{R} N_{f}-C_{A}^{2}\right)}{9 C_{A}^{3}} l^{2}\right)
\end{aligned}
$$

where $\alpha_{\mathrm{S}}=\alpha_{\mathrm{S}}(k)$, and finally,

$$
\begin{aligned}
& I\left(k^{2} ; e^{-l} k^{2}\right) \approx \frac{\alpha_{\mathrm{S}}}{2 \pi}\left(\frac{1}{2} l^{2}\right) \\
& \quad+\left(\frac{\alpha_{\mathrm{S}}}{2 \pi}\right)^{2}\left(\frac{1}{24} C_{A} l^{4}+\frac{1}{12} b l^{3}-\frac{2\left(C_{A}-C_{F}\right) T_{R} N_{f}\left(11 C_{A}^{2}-4 C_{F} T_{R} N_{f}\right)}{9 C_{A}^{3}} l^{2}\right),
\end{aligned}
$$

where again $\alpha_{\mathrm{S}}=\alpha_{\mathrm{S}}(k)$. 
Note that, in the above, the coefficients of $\alpha_{S}^{2} L^{2}$ and $\alpha_{S}^{2} l^{2}$ are not believed to be correct, since they are only next-to-next-to-leading logarithms. Nevertheless, they must be considered when performing the matching since the fixed-order result is exact to order $\alpha_{\mathrm{S}}^{2}$. It only remains to write the various $\alpha_{\mathrm{S}}$ values in terms of $\alpha_{\mathrm{S}}(Q)$ :

$$
\begin{aligned}
\frac{\alpha_{\mathrm{S}}(k)}{2 \pi} & \approx \frac{\alpha_{\mathrm{S}}}{2 \pi}+\frac{1}{2} b\left(\frac{\alpha_{\mathrm{S}}}{2 \pi}\right)^{2} \log \frac{Q^{2}}{k^{2}}, \\
\frac{\alpha_{\mathrm{S}}\left(k^{2} / Q\right)}{2 \pi} & \approx \frac{\alpha_{\mathrm{S}}}{2 \pi}+b\left(\frac{\alpha_{\mathrm{S}}}{2 \pi}\right)^{2} \log \frac{Q^{2}}{k^{2}} .
\end{aligned}
$$

The result of this expansion is most easily written in the form of Eqs. (10) and (24), with the additional notation:

$$
\begin{aligned}
\widetilde{R}\left(\tau, y_{\text {cut }}\right) & =R_{3}\left(\tau, y_{\text {cut }}\right)+2 R_{4}\left(\tau, y_{\text {cut }}\right) \\
& =\frac{\alpha_{\mathrm{S}}(Q)}{2 \pi} \widetilde{A}\left(\tau, y_{\text {cut }}\right)+\left(\frac{\alpha_{\mathrm{S}}(Q)}{2 \pi}\right)^{2}\left(\widetilde{B}\left(\tau, y_{\text {cut }}\right)-\frac{3}{2} C_{F} \widetilde{A}\left(\tau, y_{\text {cut }}\right)\right) .
\end{aligned}
$$

Then,

$$
\begin{aligned}
A\left(e^{-L}\right)= & 2 C_{F} L^{2}-3 C_{F} L \\
B\left(e^{-L}\right)= & -2 C_{F}^{2} L^{4}+C_{F}\left(6 C_{F}+b\right) L^{3}-C_{F}\left(\frac{3}{4} b+\frac{3}{2} C_{F}\right) L^{2}-\frac{9}{2} C_{F}^{2} L \\
\widetilde{A}\left(e^{-L}, e^{-l-L}\right)= & \left(2 C_{F} l^{2}-3 C_{F} l\right) \Theta(l)-C_{F}(l-L)^{2} \Theta(l-L) \\
\widetilde{B}\left(e^{-L}, e^{-l-L}\right)= & {\left[\left(\frac{1}{2} b L-\left(2 C_{F} L^{2}-3 C_{F} L\right)+\frac{3}{2} C_{F}\right)\left(2 C_{F} l^{2}-3 C_{F} l\right)\right.} \\
& +\frac{1}{6} C_{F} C_{A} l^{4}+\left(\frac{1}{3} b-\frac{1}{2} C_{A}\right) C_{F} l^{3} \\
& \left.-\frac{8\left(C_{A}-C_{F}\right) C_{F} T_{R} N_{f}\left(6 C_{A}^{2}+C_{A} T_{R} N_{f}-6 C_{F} T_{R} N_{f}\right) l^{2}}{9 C_{A}^{3}}\right] \Theta(l) \\
& -\left[\left(b L-\left(2 C_{F} L^{2}-3 C_{F} L\right)+\frac{3}{2} C_{F}\right) C_{F}(l-L)^{2}\right. \\
& +\frac{1}{12} C_{F} C_{A}(l-L)^{4}+\frac{1}{6} b C_{F}(l-L)^{3} \\
& \left.-\frac{4\left(C_{A}-C_{F}\right) C_{F} T_{R} N_{f}\left(11 C_{A}^{2}-4 C_{F} T_{R} N_{f}\right)(l-L)^{2}}{9 C_{A}^{3}}\right] \Theta(l-L) .
\end{aligned}
$$

These have been checked by solving the evolution equation iteratively, verifying that the claimed solution does actually satisfy the evolution equation, at least to second order in $\alpha_{\mathrm{S}}$.

\section{Numerical Results}

Combining the fixed-order and resummed results, we obtain the contribution to the mean jet multiplicity from events with thrust between $T$ and $T+d T, n\left(1-T, y_{\text {cut }}\right) d T$. 


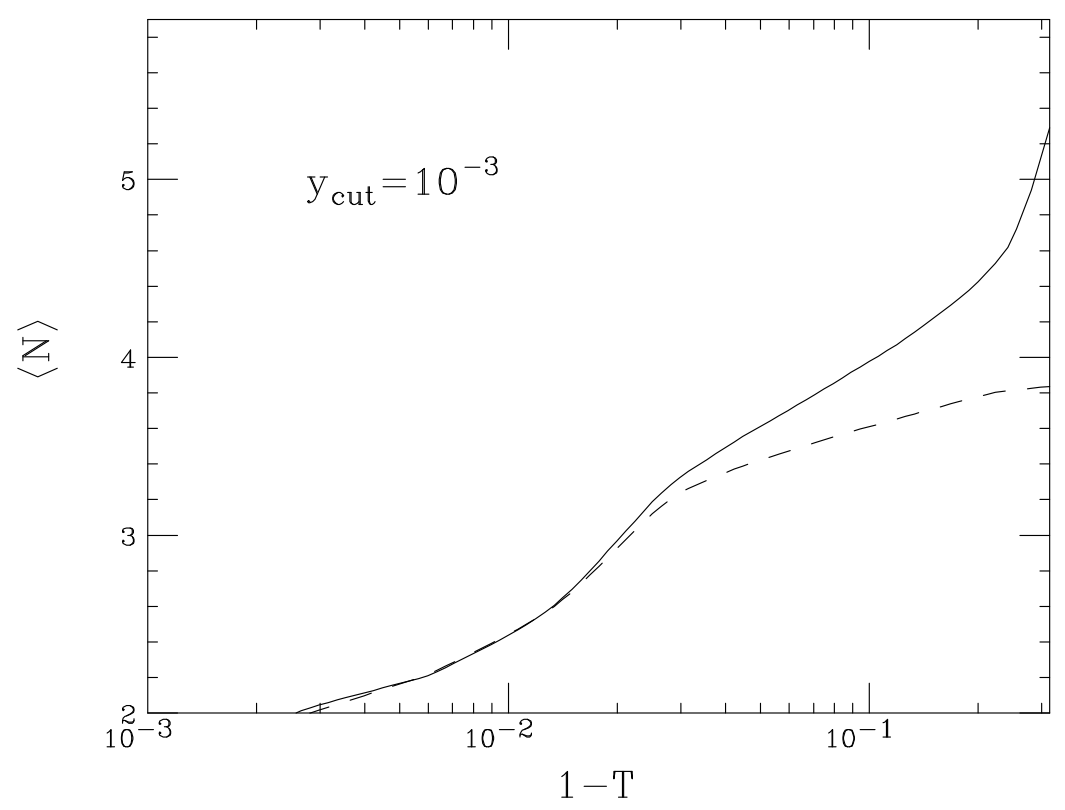

Figure 1: The mean number of jets in $e^{+} e^{-}$annihilation as a function of thrust, using the $\mathcal{O}\left(\alpha_{\mathrm{S}}^{2}\right)$ result alone (dashed) and matched with the resummed result (solid).

To obtain the mean jet multiplicity as a function of thrust we normalise this to the number of events in the same range, $r(1-T) d T$, to give

$$
\langle N\rangle(T)=\frac{n\left(1-T, y_{\text {cut }}\right)}{r(1-T)}
$$

shown in Fig. 1 for a representative value of $y_{\text {cut }}=10^{-3}$. We fix $\mu=\sqrt{s}=m_{z}$ and $\alpha_{\mathrm{S}}\left(m_{Z}\right)=0.120$. We see that for $1-T \gg y_{\text {cut }}$, the resummation is extremely important. For $1-T \sim y_{\text {cut }}$, the physical threshold, the NLO corrections are negative and even after resumming large logarithms to all orders, give an unphysical result, with the mean number of jets falling below 2 . This region is anyway outside perturbative control, since it corresponds to $k^{2}=s(1-T) \sim \Lambda \sqrt{s}$.

In Fig. 2 we show the result for fixed thrust $T=0.95$ as a function of $y_{\text {cut }}$. The resummation is again seen to be important for $y_{\text {cut }} \ll 1-T$.

The thrust can be used to separate two-jet from three-jet events. In Fig. 3 we show the multiplicity of jets in each sample. These are defined by

$$
\langle N\rangle(T>t)=\frac{N\left(1-t, y_{\text {cut }}\right)}{R(1-t)},
$$

and

$$
\langle N\rangle(T<t)=\frac{N\left(y_{\text {cut }}\right)-N\left(1-t, y_{\text {cut }}\right)}{1-R(1-t)},
$$

respectively. They can be directly compared with the results of Ref. [9], where the separation into two- and three-jet events was made using the same jet algorithm as the one in which the jets were counted - the $k_{\perp}$ algorithm. Note that for small $y_{\text {cut }}$ the $\mathcal{O}\left(\alpha_{\mathrm{S}}^{2}\right)$ results appear to be unphysical with the multiplicity in two-jet events being 


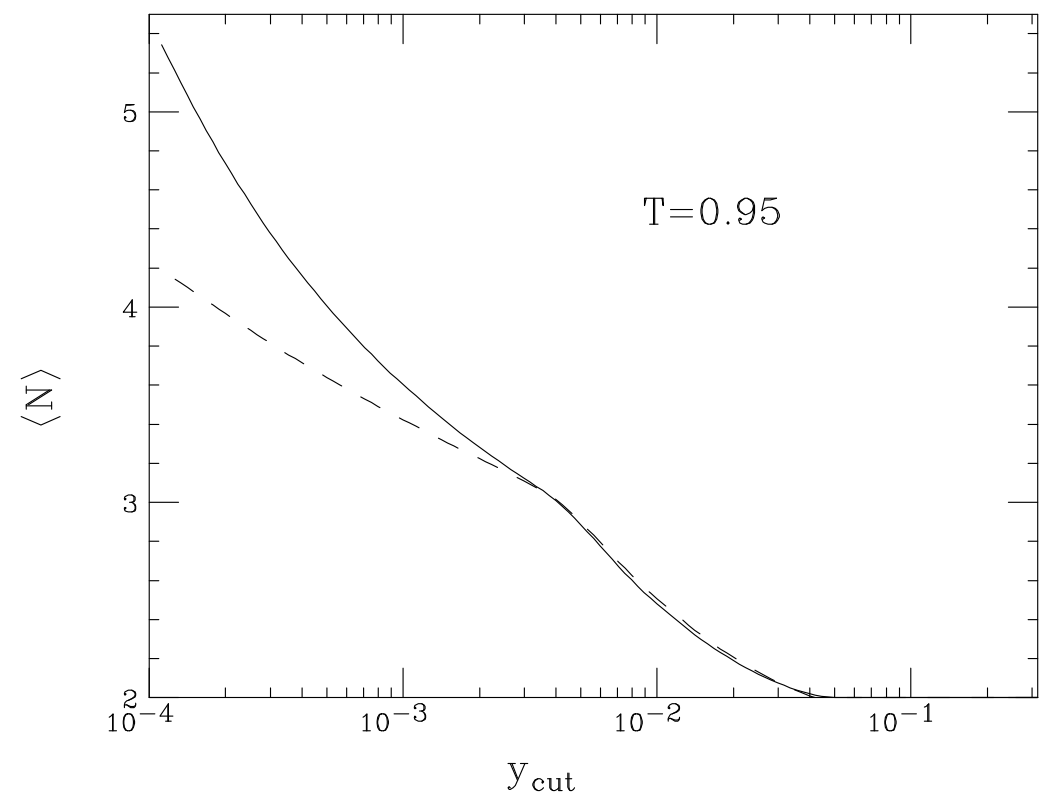

Figure 2: The mean number of jets in $e^{+} e^{-}$annihilation as a function of $y_{\text {cut }}$ for fixed thrust, using the $\mathcal{O}\left(\alpha_{\mathrm{S}}^{2}\right)$ result alone (dashed) and matched with the resummed result (solid).

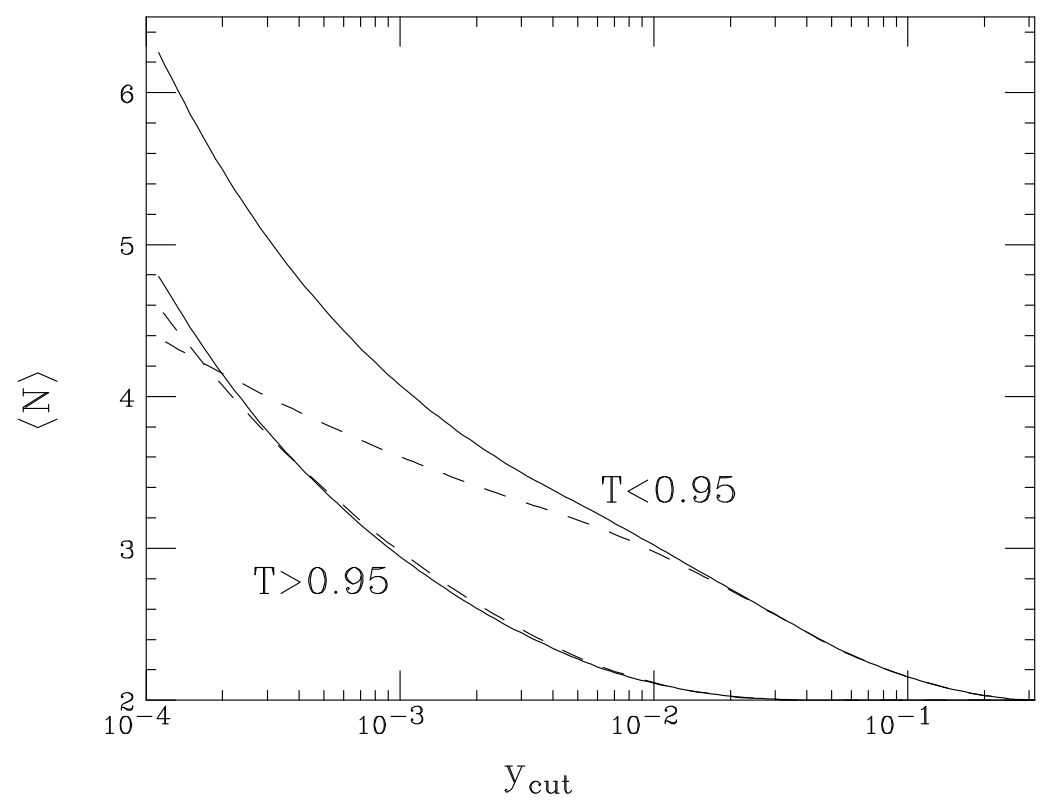

Figure 3: The mean number of jets in $e^{+} e^{-}$annihilation as a function of $y_{\text {cut }}$ for events with thrust above and below a fixed cut, using the $\mathcal{O}\left(\alpha_{\mathrm{S}}^{2}\right)$ result alone (dashed) and matched with the resummed result (solid). 


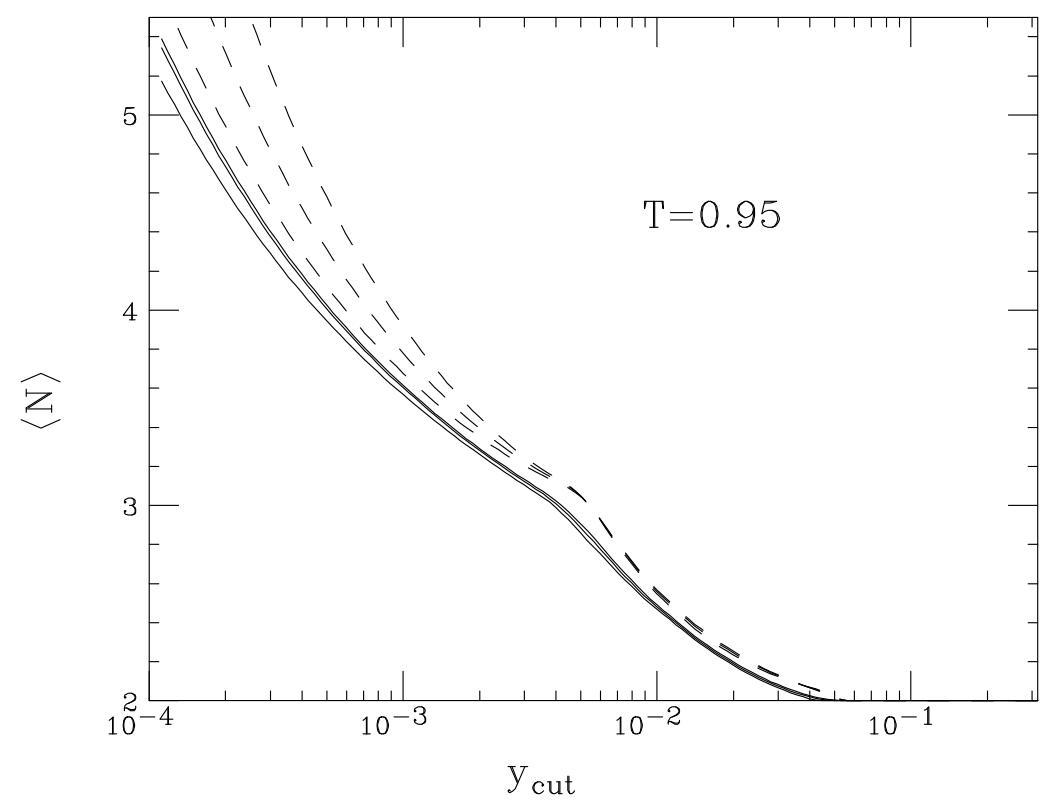

Figure 4: The mean number of jets in $e^{+} e^{-}$annihilation as a function of $y_{\text {cut }}$ for fixed thrust, using the resummed result matched with the $\mathcal{O}\left(\alpha_{\mathrm{S}}\right)$ (dashed) and $\mathcal{O}\left(\alpha_{\mathrm{S}}^{2}\right)$ (solid) results, for renormalization scale choices $\mu=\sqrt{s} / 2, \sqrt{s}$ and $2 \sqrt{s}$.

higher than for three-jet events. This is somewhat misleading as they are effectively calculated to different orders owing to the different denominators in Eqs. (74) and (75),

$$
\begin{aligned}
\langle N\rangle(T>t) & \sim \frac{2+\mathcal{O}\left(\alpha_{\mathrm{S}}\right)+\mathcal{O}\left(\alpha_{\mathrm{S}}^{2}\right)}{1+\mathcal{O}\left(\alpha_{\mathrm{S}}\right)+\mathcal{O}\left(\alpha_{\mathrm{S}}^{2}\right)} \sim 2+\mathcal{O}\left(\alpha_{\mathrm{S}}\right)+\mathcal{O}\left(\alpha_{\mathrm{S}}^{2}\right), \\
\langle N\rangle(T<t) & \sim \frac{\mathcal{O}\left(\alpha_{\mathrm{S}}\right)+\mathcal{O}\left(\alpha_{\mathrm{S}}^{2}\right)}{\mathcal{O}\left(\alpha_{\mathrm{S}}\right)+\mathcal{O}\left(\alpha_{\mathrm{S}}^{2}\right)} \sim 3+\mathcal{O}\left(\alpha_{\mathrm{S}}\right) .
\end{aligned}
$$

The all-orders results are physically behaved, because the first uncalculated term in either result is suppressed by at least two orders of $\alpha_{\mathrm{S}}$ and two powers of $\log \left(y_{\mathrm{cut}}\right)$.

One might naïvely expect that the number of jets in a three-jet event be larger than in a two-jet event by a factor $\left(2 C_{F}+C_{A}\right) /\left(2 C_{F}\right) \approx 2$, which is certainly not the case in Fig. (3) where for small $y_{\text {cut }}$ it is around 1.3. This is comparable to the ratio found in the $k_{\perp}$ algorithm of around 1.4 and, as discussed in Ref. [9] is because the form factor suppression for the gluon jet is always stronger than for the quark jets.

Finally, in Fig. 1 we show the variation of the result at fixed thrust under changes of renormalization scale between $\sqrt{s} / 2$ and $2 \sqrt{s}$. We see that the $\mathcal{O}\left(\alpha_{\mathrm{S}}^{2}\right)$ result matched with the all-orders resummation is considerably more stable than the $\mathcal{O}\left(\alpha_{\mathrm{S}}\right)$ plus resummed result. 


\section{References}

1. S. Catani, Yu.L. Dokshitzer, M. Olssen, G. Turnock and B.R. Webber, Nucl. Phys. B269 (1991) 432

2. S. Catani, Yu.L. Dokshitzer, F. Fiorani and B.R. Webber, Nucl. Phys. B377 (1992) 445

3. B.R. Webber 'Renormalon Phenomena in Jets and Hard Processes', talk given at 27th International Symposium on Multiparticle Dynamics (ISMD 97), Frascati, Italy, 8-12 Sep 1997, hep-ph/9712236

4. S. Catani, L. Trentadue, G. Turnock and B.R. Webber, Nucl. Phys. B407 (1993) 3

5. See for example M. Schmelling, Phys. Scr. 51 (1995) 683 and references therein

6. K. Kimura and K. Tesima, Zeit. Phys. C62 (1994) 471

7. N. Brown and W. J. Stirling, Zeit. Phys. C53 (1992) 629

8. S. Catani and M.H. Seymour, Phys. Lett. 378B (1996) 287; Nucl. Phys. B485 (1997) 291 and erratum ibid. 510 (1997) 503

9. S. Catani, Yu.L. Dokshitzer, F. Fiorani and B.R. Webber, Nucl. Phys. B383 (1992) 419 